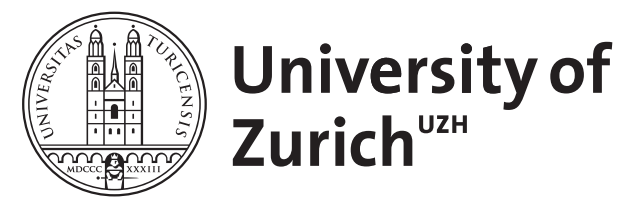

\title{
Säuglingsschreien und Schlaf-Wach-Regulation
}

\author{
Jenni, O G
}

\begin{abstract}
Schreien ist eine vorsprachliche Kommunikationsart, mit welcher der Säugling seine Bedürfnisse und seine Befindlichkeit äußert. In den ersten Lebensmonaten schreien Säuglinge allerdings häufig, ohne dass es Eltern und Fachleuten gelingt, einen Grund dafür zu finden. Der vorliegende Artikel stellt ein Erklärungsmodell für dieses so genannte unspezifische Schreien vor, das einen erhöhten Wachheitsgrad widerspiegelt, der von der inneren Uhr gesteuert wird und welchem die Schlafhomöostase wegen noch fehlender oder verzögerter Reifungsentwicklung nicht entgegenwirkt. Der Säugling zeigt darum im Verlauf des Tages eine Überreizung und einen besonders intensiven Wachzustand, sodass er nicht abschalten kann, visuelle und vestibuläre Reize sucht und nicht in der Lage ist, sich selber zu beruhigen. Erst das Einsetzen der Schlafhomöostase mit 6 Wochen führt dazu, dass das Schreien des Säuglings abnimmt. Eine Rhythmisierung von Schlaf- und Wachphasen, eine Reizreduktion und individuell abgestimmte Beruhigungsstrategien können die Schreidauer reduzieren.
\end{abstract}

DOI: https://doi.org/10.1007/s00112-008-1891-8

Other titles: Early infant crying and sleep-wake regulation

Posted at the Zurich Open Repository and Archive, University of Zurich ZORA URL: https://doi.org/10.5167/uzh-28809

Journal Article

Published Version

Originally published at:

Jenni, O G (2009). Säuglingsschreien und Schlaf-Wach-Regulation. Monatsschrift Kinderheilkunde, 157(6):551-557.

DOI: https://doi.org/10.1007/s00112-008-1891-8 
Monatsschr Kinderheilkd 2009 • 157:551-557 DOI 10.1007/s00112-008-1891-8

Online publiziert: 14. Mai 2009

๑) Springer Medizin Verlag 2009

Redaktion

M. Papoušek, München

D. Reinhardt, München
O. Jenni

Abteilung Entwicklungspädiatrie, Universitätskinderkliniken Zürich

\begin{abstract}
Schreien ist eine vorsprachliche Kommunikationsart, mit welcher der Säugling seine Bedürfnisse und seine Befindlichkeit äußert. Der Säugling schreit, wenn er Hunger, nasse Windeln oder Schmerzen hat, wenn er von Sinnesreizen überflutet wird oder weil er nicht alleine sein will. In den ersten Lebensmonaten schreien Säuglinge allerdings häufig, ohne dass es Eltern gelingt, einen Grund dafür zu finden. Es gibt eine Reihe von Hypothesen zur Ursache des so genannten unspezifischen Schreiens, das auch als exzessives Schreien bezeichnet wird, wenn es für die Eltern ein tolerierbares Maß überschreitet. Dieser Artikel stellt ein Erklärungsmodell zu dessen Entstehung vor.
\end{abstract}

\section{Säuglingsschreien}

Häufig kommen verzweifelte Eltern mit ihrem schreienden Säugling in die Notaufnahme eines Kinderspitals oder konsultieren den Kinderarzt in der Praxis. Laut epidemiologischen Studien leidet jede 5. Familie in den ersten Lebensmonaten unter dem Schreiverhalten ihres Säuglings [15, 24]. Die Aufgaben des Kinderarztes bestehen zunächst darin, mittels Anamnese und klinischer Untersuchung zwischen verschiedenen Schreiarten zu unterscheiden und allenfalls diagnostische und therapeutische Maßnahmen zu erwägen, wenn dem Schreiverhalten des Kindes eine organische Störung zugrunde liegt.

Aus entwicklungspädiatrischer Sicht werden 3 Schreiarten des jungen Säuglings unterschieden.

1. Physiologisches Schreien nach der Geburt, bei Hunger, nassen Windeln oder emotionalen Bedürfnissen (z. B. nach Zuwendung der Bezugsperson)

2. Pathologisches Schreien wegen einer akuten Erkrankung mit Schmerzen oder Unwohlsein (z. B. Ösophagitis bei gastroösophagealem Reflux, Gastroenteritis oder Infekt der oberen Luftwege), einer chronischen Erkrankung (z. B. Nahrungsmittelallergie oder hypoxisch-ischämischer Enzephalopathie) oder einer genetischen Störung (z. B. Katzenschreisyndrom). Dem pathologischen Schreien liegt immer eine organische Ursache zugrunde.

3. Unspezifisches Schreien, das bei fast allen Säuglingen vorkommt und bei welchem Eltern und Fachleute keine unmittelbare Ursache finden

\section{Unspezifisches und exzessives Schreien}

Überschreitet das Ausmaß des unspezifischen Schreiens ein für die Eltern tolerierbares Maß, wird der Begriff exzessives Schreien verwendet. Für wissenschaftliche Studien wird gewöhnlich die Dreierregel von Wessel et al. [26] als Definition des exzessiven Schreiens angewandt: Ein gesunder Säugling zeigt Unruhe, Quengeln oder Schreien

1. während mehr als $3 \mathrm{~h} / \mathrm{Tag}$,

2. an mehr als 3 Tagen/Woche und

3. seit mehr als 3 Wochen.

Der Begriff „exzessiv“ orientiert sich in dieser Beschreibung an einer messbar erhöhten Schreidauer und Frequenz. Diese Kriterien mögen für eine zuverlässige Vergleichbarkeit von Forschungsergebnissen sinnvoll sein, sind im klinischen Alltag aber wenig hilfreich. Es gibt nicht selten Eltern, die ih- re Kinder während weniger als $3 \mathrm{~h} / \mathrm{Tag}$ und an weniger als 3 Tagen/Woche als exzessive Schreikinder betrachten (• Abb. 1, [24]). Darum muss der Belastungsgrad der Bezugspersonen bei der Diagnose des exzessiven Schreiens berücksichtigt werden.

\section{(7) Die Dreierregel ist im klinischen Alltag wenig hilfreich}

Es gibt unzählige Erklärungsversuche zum unspezifischen und exzessiven Schreien. So prägten Wessel et al. [26] den Begriff Kolik, weil man davon ausging, dass das Schreien infolge einer gastrointestinalen Störung mit Krämpfen und Blähungen entsteht. Der Begriff wird nach wie vor häufig gebraucht, obwohl Studien zeigten, dass Verdauungsstörungen nur in wenigen Fällen als Ursache für das Säuglingsschreien anzusehen sind [16]. Organische Gründe für ein ausgeprägtes Schreien sind z. B. eine Kuhmilchproteinintoleranz, gastroösophagealer Reflux, Gastroenteritis mit Invagination, Hodentorsion, Otitis media oder viele andere Erkrankungen, bei welchen das Schreien des Kindes primär Ausdruck der empfundenen Schmerzen ist (pathologisches Schreien). Das Schreien zeigt in diesen Fällen nicht die typischen Merkmale des unspezifischen Säuglingsschreiens.

\section{Merkmale des unspezifischen Säuglingsschreiens}

Entwicklungsverlauf. Das Schreiverhalten von Säuglingen zeigt einen typischen $\mathrm{n}$-förmigen Entwicklungsverlauf $[1,6]$. Schreiepisoden beginnen in der 2. Lebenswoche und nehmen an Intensität zu. Das Schreien erreicht meist im 2. Monat ein Maximum (Schreigipfel mit 6 Wo- 


\section{Leitthema}
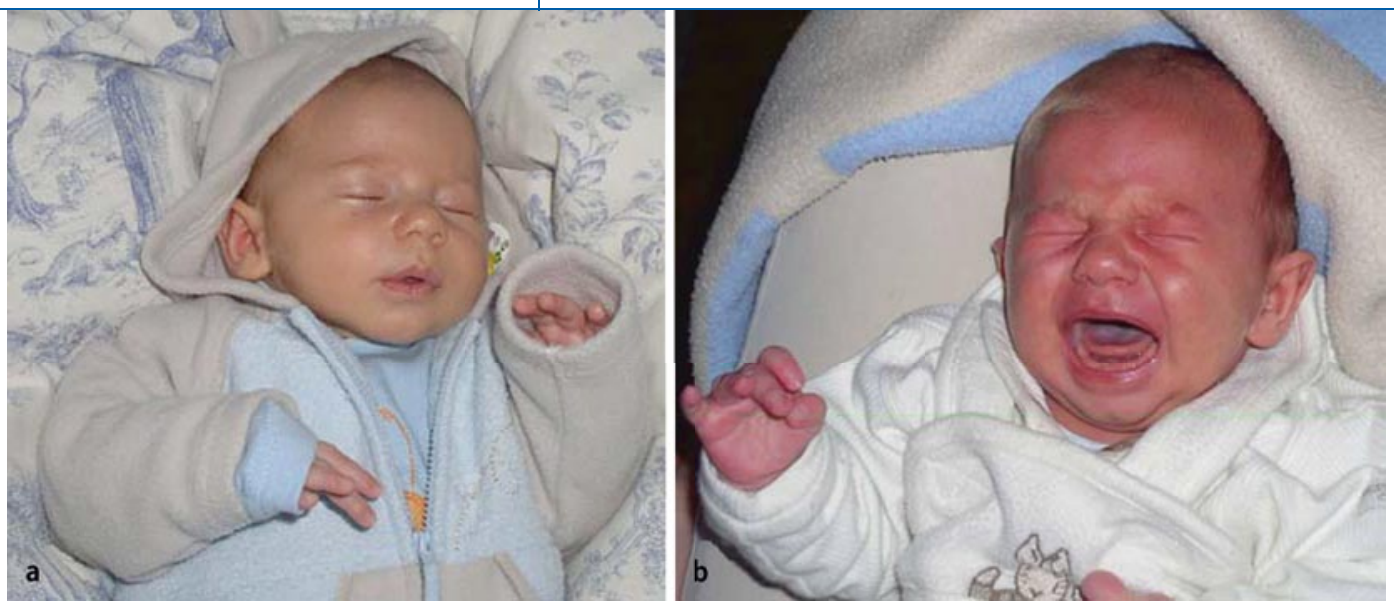

Abb. $1 \varangle$ Schlafendes (a) und schreiendes (b) Kind

chen) und geht danach bis zum 3. Monat wieder zurück (• Abb. 2).

Tagesverlauf. Das Schreien zeigt einen typischen tageszeitlichen Verlauf. Schreiepisoden sind in der Nacht und am Morgen selten $[1,6]$. Sie treten v. a. in den Abendstunden auf (abendlicher Schreigipfel zwischen 16 und 22 Uhr, $\bullet$ Abb. 3).

Interindividuelle Variabilität. Die Unterschiede in der Schreidauer sind zwischen Kindern sehr groß [1]. Im Alter von 6 Wochen schreien die einen Säuglinge knapp $1 \mathrm{~h}$, andere über $3 \mathrm{~h}$. Kinder zeigen sehr unterschiedliche Schreikurven, manche erreichen den Schreigipfel bereits in der 3. Lebenswoche, andere erst in der 8 . Woche.

Barr [2] postulierte, dass sich exzessives Schreien in der Qualität nicht vom unspezifischen Schreien unterscheide, sondern es einzig eine extreme Ausprägung der normalen individuellen Variabilität widerspiegle. Mit anderen Worten: Schreikinder schreien nicht anders als gesunde Säuglinge, sie schreien einfach mehr.

Soziodemografische Faktoren. Geburtsrang, sozioökonomischer Status, Bildungsstand der Eltern oder elterliche Erfahrung und Pflegeverhalten haben keinen wesentlichen Einfluss auf das Schreiverhalten der Säuglinge [4].

\section{Schreiverhalten in traditionellen Kultu-}

ren. Der typische Entwicklungsverlauf des Säuglingsschreiens wurde in verschiedenen traditionellen Kulturen wie den Kung in Botswana nachgewiesen [3], wenn auch mit gewissen Variationen (z. B. einem abgeschwächten Schreigipfel mit kürzerer Schreidauer).
Besonders der typische Entwicklungsund Tagesverlauf in westlichen und traditionellen Kulturen deuten auf ein universelles biologisches Phänomen hin. Dieser Umstand wird durch den geringen Einfluss von soziodemografischen Faktoren auf das Schreiverhalten bestätigt. Man ist heute weitgehend der Ansicht, dass das unspezifische Schreien primär ein Ausdruck von frühen physiologischen Anpassungsprozessen des zentralen Nervensystems ist [4]. Der Artikel stellt ein Erklärungsmodell dieser neuronalen Entwicklungsprozesse anhand des 2-Prozess-Modells der Schlaf-Wach-Regulation [5] vor.

\section{Innere Uhr und Schlafhomöostase im Wechselspiel}

\section{Grundlagen der Schlaf-Wach-Regulation}

Das Arbeitsmodell von Borbély [5] beschreibt 2 biologische Prozesse, welche Schlaf und Wachsein beim Menschen steuern (2-Prozess-Modell der SchlafWach-Regulation [5], • Abb.4).

1. Zirkadianer Prozess

2. Schlafhomöostase

\section{Zirkadianer Prozess}

Er beschreibt einen regelmäßigen und schlafunabhängigen Prozess, der es dem Individuum ermöglicht, nachts zu schlafen und tagsüber wach und geistig aktiv zu sein [17]. Er ist anatomisch in den suprachiasmatischen Kernen des Zwischenhirns lokalisiert (,innere Uhr") und steuert nicht nur Wachheit und Schlaf, sondern viele physiologische Prozesse wie Körpertemperatur, Atmung, Blutdruck, Herztätigkeit, Harnausscheidung, Hormonpro- duktion, Aufmerksamkeit, kognitive Leistungsfähigkeit oder Genaktivität. Die innere Uhr wird mit regelmäßig wiederkehrenden Umgebungsfaktoren wie dem 24h-Tag-Nacht-Wechsel synchronisiert.

\section{- Der wichtigste äußere \\ Zeitgeber ist das Tageslicht.}

Andere Zeitgeber wie Lärm, soziale Kontakte oder regelmäßige Nahrungsaufnahme spielen ebenfalls eine Rolle [17].

Die Steuerungsprozesse der inneren Uhr sind gewöhnlich so eingestellt, dass die Wachheit morgens am geringsten und in den Abendstunden am stärksten ist (- Abb. 4, [7]). Dieser Umstand scheint auf den ersten Blick widersprüchlich zu eigenen Beobachtungen zu sein. In Bunkerexperimenten ohne Kontakt zur Außenwelt und Wissen über die Uhrzeit konnte man aber zeigen, dass Menschen besonders in den frühen Morgenstunden viel schlafen und in den Abendstunden wach und aktiv sind [7]. Der Wachheitsgrad ist dabei eng an den Verlauf der Körpertemperatur gekoppelt: Je wacher man ist, desto höher ist die Körpertemperatur [7]. Diese Befunde führten zum Begriff der „forbidden zone for sleep" in den frühen Abendstunden. Es scheint, dass wir uns mit einer Phase hoher Aktivität und zirkadianer Wachheit am Abend auf die Nacht vorbereiten. Würden wir tatsächlich in den frühen Abendstunden ein Nickerchen machen, würde sich der Einschlafzeitpunkt weit in die Nacht verschieben und brächte den Schlaf-WachRhythmus völlig durcheinander.

Dass wir trotz geringer zirkadianer Einschlafbereitschaft in den frühen Abendstunden etwas später doch einschlafen können oder uns trotz hoher zirkadianer 
Müdigkeit am Morgen nicht gleich wieder ins Bett legen, bewirken der homöostatische Prozess und dessen Wechselspiel mit dem zirkadianen System.

\section{Schlafhomöostase}

Es handelt sich um einen schlafabhängigen Prozess [5]. Während des Wachseins häuft sich eine Schlafschuld an, d. h. die homöostatische Einschlafbereitschaft und der Schlafdruck nehmen im Verlauf des Tages so weit zu, dass wir schließlich einschlafen (- Abb. 4). Der abendliche Schlafdruck wird im Verlauf der Nacht wieder abgebaut. Je länger wir wach sind, desto größer sind die Schlafschuld und damit die homöostatische Einschlafbereitschaft und desto tiefer und länger schlafen wir.

Eine anatomische Lokalisation der Schlafhomöostase wie bei der inneren Uhr wurde bisher nicht gefunden. Es sind verschiedene neuronale Mechanismen beschrieben, welche die Funktion der Schlafhomöostase $\mathrm{zu}$ erklären versuchen. So reichert sich möglicherweise ein „Schlafstoff“ (z. B. der Neurotransmitter Adenosin [20]) während des Wachseins im Gehirn an, der im Schlaf wieder abgebaut wird. Eine andere Hypothese besagt, dass neuronale Prozesse im Wachzustand zu einer Verstärkung von synaptischen Verbindungen im Gehirn führen und dass der Schlaf diese Verstärkungen wieder auf ein energetisch tragbares Niveau abschwächt, was für Lernen und Gedächtnis notwendig ist (homöostatische Regulation der Synapsenfunktion, „synaptic sleep homeostasis“ [25]).

\section{Wechselwirkungen im 2-Prozess-Modell}

Idealerweise sind der homöostatische und der zirkadiane Prozess aufeinander abgestimmt. Edgar et al. [8] prägten für das Wechselspiel der beiden Prozesse den Begriff „opponent processes“: Der geringe Schlafdruck nach dem Aufwachen kompensiert die hohe zirkadiane Müdigkeit in den frühen Morgenstunden, und der im Verlauf des Tages zunehmende Schlafdruckgleicht das zirkadiane Aktivitätsmaximum und die Wachheit am Abend aus.

Die optimale Abstimmung der beiden Prozesse ist eine Voraussetzung für einen stabilen und aufmerksamen Verhaltenszustand am Tag und einen ruhigen Schlaf in der Nacht. Eine fehlende oder abnorme

Monatsschr Kinderheilkd 2009 · 157:551-557 DOI 10.1007/s00112-008-1891-8

○) Springer Medizin Verlag 2009

O. Jenni

Säuglingsschreien und Schlaf-Wach-Regulation

Zusammenfassung

Schreien ist eine vorsprachliche Kommunikationsart, mit welcher der Säugling seine Bedürfnisse und seine Befindlichkeit äußert. In den ersten Lebensmonaten schreien Säuglinge allerdings häufig, ohne dass es Eltern und Fachleuten gelingt, einen Grund dafür zu finden. Der vorliegende Artikel stellt ein Erklärungsmodell für dieses so genannte unspezifische Schreien vor, das einen erhöhten Wachheitsgrad widerspiegelt, der von der inneren Uhr gesteuert wird und welchem die Schlafhomöostase wegen noch fehlender oder verzögerter Reifungsentwicklung nicht entgegenwirkt. Der Säugling zeigt darum im Verlauf des Tages eine Überreizung und einen besonders intensiven Wachzustand, sodass er nicht abschalten kann, visuelle und vestibuläre Reize sucht und nicht in der Lage ist, sich selber zu beruhigen. Erst das Einsetzen der Schlafhomöostase mit 6 Wochen führt dazu, dass das Schreien des Säuglings abnimmt. Eine Rhythmisierung von Schlaf- und Wachphasen, eine Reizreduktion und individuell abgestimmte Beruhigungsstrategien können die Schreidauer reduzieren.

Schlüsselwörter Exzessives Schreien · Regulationsstörung · Säuglingskoliken · Schlafhomöostase .

Zirkadiane Regulation

\section{Early infant crying and sleep-wake regulation}

\section{Abstract}

Crying in the first weeks of life is an honest signal of infant needs. However, young infants often cry without obvious reasons. This article presents an explanatory model for early infant crying, which may reflect an interaction between the increased circadian alertness governed by the biologic clock and the delayed maturation of sleep homeostatic mechanisms. As a consequence, crying infants show increasing hyperalertness and sensory overload at the end of the day, search intensively for visual and vestibular stimuli, and are unable to soothe themselves. When sleep homeostatic processes appear at age 6 weeks, crying duration decreases. A regular sleep-wake schedule, a reduction of sensory stimuli, and individual soothing strategies may reduce infant crying in the first months of life.

\section{Keywords}

Excessive infant crying · Infant colic · Sleepwake regulation - Sleep homeostasis - Circadian rhythms 


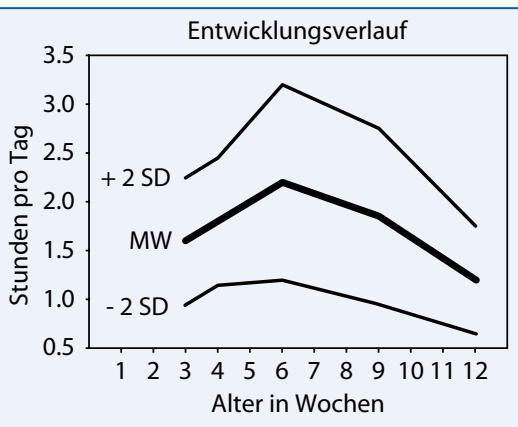

Abb. $2 \Delta$ Entwicklungsverlauf des unspezifischen Schreiens, MW Mittelwert, SD Standardabweichung. (Nach [1])

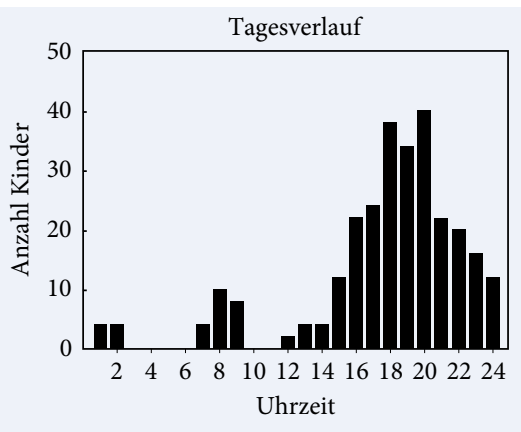

Abb. $3 \Delta$ Tagesverlauf des unspezifischen Schreiens im Alter von 6 Wochen $(n=280)$. (Nach [6])

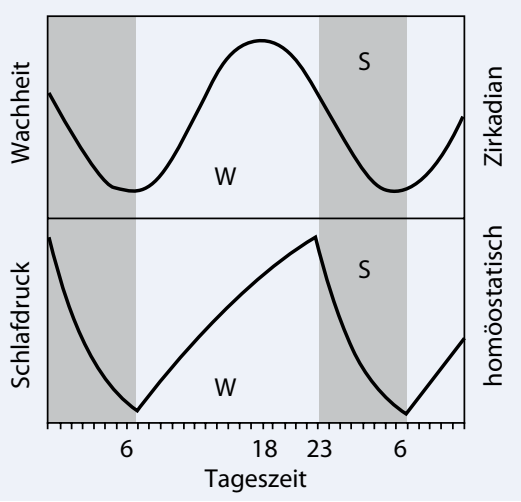

Abb. $4 \Delta$ 2-Prozess-Modell der Schlaf-Wach-Regulation, WWachsein, S Schlaf. (Nach [5])

Abstimmung der beiden Prozesse führt zu Einschlafschwierigkeiten, Durchschlafproblemen, vermehrter Tagesmüdigkeit oder Störungen in der Verhaltensregulation. Ein bekanntes Bespiel dafür ist der Jetlag, bei welchem nach einem Langstreckenflug über mehrere Zeitzonen die innere Uhr und die Schlafhomöostase nicht mehr aufeinander abgestimmt sind.

\section{EntwicklungderSchlaf-Wach-Regula- tion in den ersten Lebensmonaten}

Zirkadianer und homöostatischer Prozess durchlaufen in den ersten Lebensmona- ten Reifeveränderungen und stimmen ihre Funktionen aufeinander ab [12]. Emde et al. [9] bezeichneten diese Entwicklungsveränderungen als „biobehavioral shift“. Man kann davon ausgehen, dass die Entwicklung sowie das Wechselspiel der inneren Uhr und der Schlafhomöostase ursächlich mit dem Schlaf- und Schreiverhalten der ersten Lebensmonate zusammenhängen.

\section{- Das zirkadiane System ist im letzten Trimester der Schwangerschaft bereits funktionstüchtig.}

Verschiedene Studien bei Primaten und Menschen ergaben, dass Herzfrequenz, Körpertemperatur und Hormonausscheidung bereits intrauterin einen 24-h-Rhythmus zeigen, der durch die mütterlichen Zeitgeber synchronisiert wird [22]. Die Amplitude dieser 24-h-Rhythmen wird im Verlauf der ersten Lebenswochen immer größer, d. h. die Stärke des zirkadianen Signals nimmt immer mehr zu [14]. So erreicht z. B. die Amplitude des Temperaturrhythmus im Alter von 6-12 Wochen bereits ein Maximum. Weil die Körpertemperatur mit dem Aktivitätsgrad und der Wachheit eines Individuums eng gekoppelt ist [7], kann man davon ausgehen, dass mit zunehmender Amplitude des Temperaturrhythmus auch die Aktivität und Wachheit (,alertnes“") im Verlauf der ersten Lebenswochen immer größer werden (• Abb. 5).

\section{( Die homöostatische Schlaf-Wach-Regulation setzt erst im 2. Monat ein}

Experimentelle Studien wiesen darauf hin, dass die homöostatische Schlaf-Wach-Regulation im Gegensatz zum zirkadianen Prozess erst später auftritt [13]. Mit anderen Worten: Neugeborene und junge Säuglinge bauen noch keine Schlafschuld während des Wachseins auf und kompensieren Wachzeit nicht mit tiefem oder längerem Schlaf. Dieser Befund deckt sich mit der elterlichen Beobachtung, dass Neugeborene nach einer längeren Wachphase manchmal nur für kurze Zeit schlafen und nachher wieder wach und aktiv sind. Deshalb hat ein längeres Wachhalten von Säuglingen keine längere Schlafphase danach zur Folge. Das Einsetzen der homöostatischen Regulation von Schlaf- und Wachphasen im
2. Lebensmonat führt dazu, dass Säuglinge längere Phasen von aufmerksamen Wachzuständen tagsüber und ruhigen Schlafphasen in der Nacht aufrechterhalten können. Die Entwicklung der Schlafhomöostase spiegelt sich neurophysiologisch im Auftreten von Deltaschlaf und Schlafspindeln im Alter zwischen 6 und 12 Wochen wider [13]. Je weiter die Reifungsprozesse der Schlafhomöostase fortschreiten, desto weniger schlafen die Kinder tagsüber und desto seltener erwachen sie in der Nacht. Die Fähigkeit, längere Phasen eines aufmerksamen Wachzustandes aufrechtzuerhalten, ist ein Zeichen der fortschreitenden Hirnreifung und führt dazu, dass Entwicklungsaufgaben in den ersten Lebensmonaten überhaupt möglich sind, z. B. exploratives Verhalten und zielgerichtete Interaktionen mit Bezugspersonen.

\section{Säuglingsschreien und Entwick- lung der Schlaf-Wach-Regulation}

Schreien scheint im Kontext der SchlafWach-Regulation einen erhöhten Wachheitsgrad darzustellen, der von der inneren Uhr gesteuert wird und welchem die Schlafhomöostase wegen noch fehlender oder verzögerter Reifungsentwicklung nicht entgegenwirkt.

\section{Tagesverlauf (abendlicher Schreigipfel)}

Das abendliche Schreimaximum kann mit der zirkadianen Wachheit erklärt werden, die im Tagesverlauf zunimmt (• Abb. 5). Wie beim Erwachsenen scheinen die zirkadiane Einschlafbereitschaft und Müdigkeit von Säuglingen am Abend gering zu sein („forbidden zone for sleep“). Dieser Umstand zeigt sich auch darin, dass Säuglinge in den ersten Lebenswochen besonders am Abend motorisch sehr aktiv sind und die abendliche Herzschlagrate im Gegensatz zum Morgen deutlich erhöht ist [10]. Dieser besonders intensive Wachzustand („hyperalterness") führt zu einer Überreizung und vermehrtem Schreien. Die Säuglinge können nicht abschalten, suchen vermehrt nach visuellen und vestibulären Reizen und sind nicht in der Lage, sich selber zu beruhigen.

Es überrascht also nicht, dass sich der Höhepunkt des Schreiens am Abend fin- 
Hier steht eine Anzeige.

算 Springer 

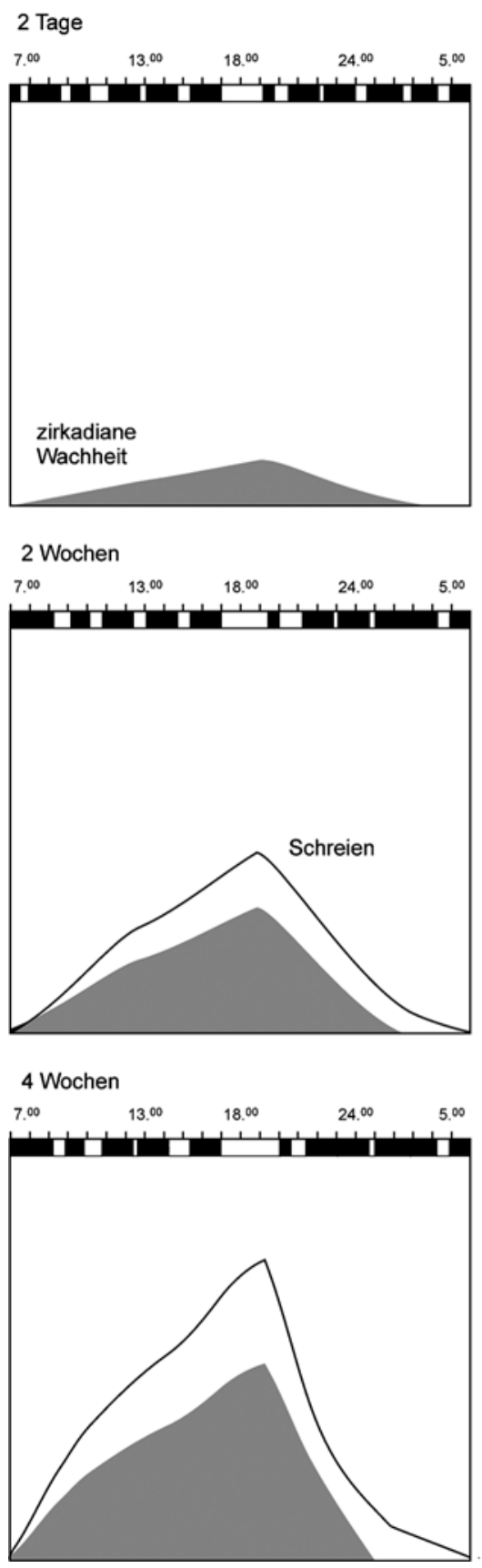

Abb. $5 \Delta$ Erklärungsmodell der zirkadianen und homöostatischen Schlaf-Wach-Regulation und des Schreiens in den ersten 12 Lebenswochen

det, besonders in den ersten Wochen, wenn die Schlafhomöostase als „opponent process" noch nicht kompensatorisch dagegen wirkt.

\section{Entwicklungsverlauf (Schreigipfel im 2. Lebensmonat)}

Das Wechselspiel der zirkadianen und der homöostatischen Schlaf-Wach-Regulation kann erklären, weshalb das Schreien in den ersten Wochen zu- und dann wieder abnimmt (• Abb. 5). Den zunehmenden zir-

6 Wochen

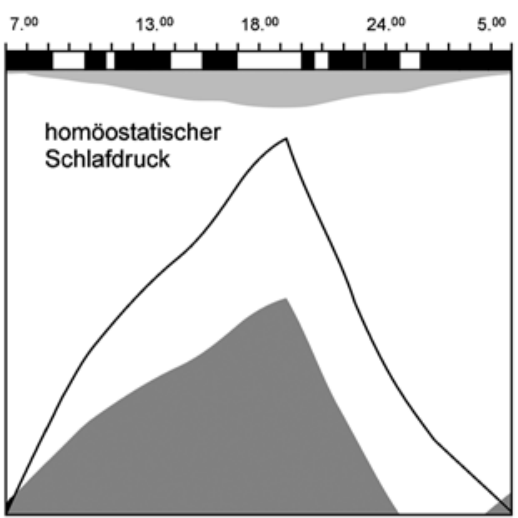

9 Wochen

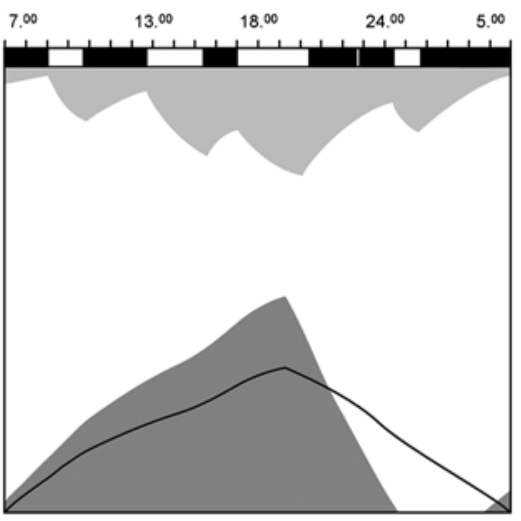

12 Wochen tagsüber ein Schlafdefizit aufweisen [4].

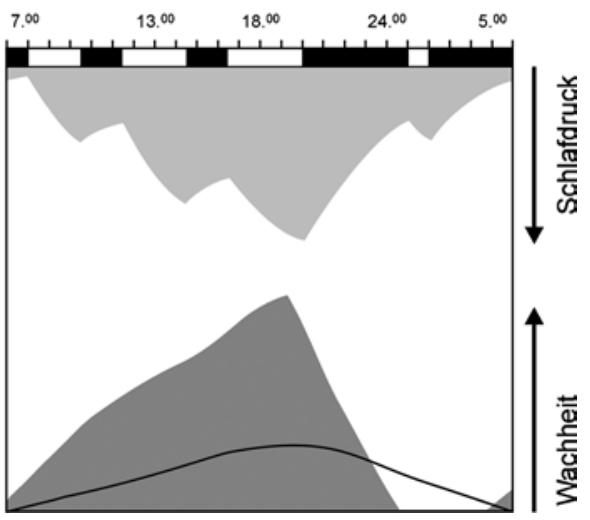

过

gens
Zeitgeber eingestellt werden, wobei soziale Rhythmen bei Säuglingen (Ernährung, Pflege und soziale Interaktion) wahrscheinlich eine größere Rolle spielen als das Tageslicht [17]. Es ist also denkbar, dass die sozialen Zeitgeber der Mutter bei häufigem Körperkontakt mit Tragen effizienter wirken, als wenn das Kind oft alleine in der Krippe liegt. Tatsächlich konnte man zeigen, dass ein konstanter Körperkontakt mit Tragen des Kindes die Schreidauer etwas reduziert [23].

\section{Persistierendes Säuglingsschreien und frühkindliche Regulationsstörung} Lebensmonat wird dem zirkadianen Signal entgegengewirkt, und das Schreien nimmt ab. Die fehlende homöostatische Regulation führt auch dazu, dass schreiende Säuglinge die vermehrten Wachphasen nicht mit Schlafen kompensieren und darum v.a.

Der Schreigipfel (je nach Kind im Alter zwischen 4 und 8 Wochen) entspricht also dem Einsetzen der homöostatischen
Bei den meisten Kindern ist das Schreien auf die ersten 3 Lebensmonate beschränkt und ohne langfristige negative Folgen. Es gibt allerdings Säuglinge, die über die ersten Monate hinaus unruhig sind, vermehrt schreien und sich ungenügend selber beruhigen können. Das Schreiverhalten ist in diesen Fällen häufig mit Durchschlaf- und Fütterungsproblemen assozi- 
iert und wird als frühkindliche Regulationsstörung bezeichnet [19]. Dieser Begriff wird verwendet, wenn das Kind älter als 3 Monate ist und sich das Schreien nicht als vorübergehendes Phänomen im Sinne eines unspezifischen Schreiens zeigt.

\section{( Kinder mit Regulationsstörung haben häufig Durchschlafprobleme}

Weil Kinder mit einer Regulationsstörung häufig Durchschlafprobleme zeigen, liegt die Vermutung nahe, dass es sich um eine Entwicklungsverzögerung der SchlafWach-Regulation handelt. Ausgehend vom 2-Prozess-Modell wird folgende These postuliert: Die Schlafhomöostase entwickelt sich bei Kindern mit persistierendem Schreien verzögert und ist mit dem zirkadianen Prozess nicht abgestimmt. Das Kind zeigt deshalb wechselnde Phasen mit Übermüdung und Überreizung, ist nicht in der Lage, einen aufmerksamen Wachzustand aufrechtzuerhalten, und erwacht mehrfach in der Nacht. Die gestörte Schlafhomöostase ist möglicherweise mit einer Reifungsverzögerung von Neurotransmitter- oder Synapsenfunktionen assoziiert $[20,25]$, die sich klinisch mit leichten neurologischen Auffälligkeiten äußert [18].

Verschiedene Autoren äußerten den Verdacht, dass Kinder mit Regulationsstörungen ein erhöhtes Risiko für langfristige Probleme der Verhaltensregulation und für Beeinträchtigungen der kognitiven Leistungsfähigkeit aufweisen $[21,27]$. Wenn die kindlichen Auffälligkeiten in der Schlaf-WachRegulation von den Eltern nicht mit Verhaltensanpassungen aufgefangen werden und Familien nicht in der Lage sind, erforderliche Hilfen zu geben, ist das Risiko für langfristige Störungen und Belastungen tatsächlich hoch. Eine mangelnde Passung zwischen elterlichem Verhalten und regulatorischen Fähigkeiten des Säuglings kann dann zu persistierenden Verhaltensauffälligkeiten und dysfunktionalen Interaktionsmustern zwischen Eltern und Kind führen [28].

\section{Fazit für die Praxis}

Die Entwicklungsberatung ist neben der Suche nach familiären Ressourcen eine wichtige Aufgabe des Kinderarztes. Es kann Eltern entlasten, wenn sie wissen, dass das Schreiverhalten ihres Säuglings Ausdruck der normalen Entwicklung der Schlaf-Wach-Regulation ist und dass keine organische Erkrankung vorliegt. Zwar findet sich sowohl in Elternratgebern als auch in der Fachliteratur eine verwirrende Vielzahl von Verhaltenstipps, aber laut den meisten wissenschaftlichen Studien haben Verhaltensänderungen oder Interventionen keine oder nur geringe Beruhigungseffekte [28], da die neuronale Reifungsentwicklung der zirkadianen und homöostatischen Schlaf-Wach-Regulation nicht wesentlich beeinflusst werden kann. Zudem kann die Wirksamkeit von Interventionen wegen der hohen Rate an Spontanremissionen in den ersten Lebensmonaten nicht zuverlässig überprüft werden. Die wichtigste Maßnahme ist die Rhythmisierung von Schlaf-, Wach- und Fütterphasen, die zur Einstellung und Stabilisierung des zirkadianen Systems beiträgt [11], welche aber zirkadiane Wachheit nur in beschränktem Maß beeinflusst. Eine weitere Empfehlung ist die Reizreduktion, besonders in den Abendstunden, wenn die zirkadiane Wachheit hoch ist. Hilfreich sind besonders Beruhigungsstrategien, die zusammen mit den Eltern erarbeitet werden. Manche Ratgeber empfehlen, das Kind einfach schreien zu lassen. Diese Maßnahme ist aber nicht angemessen, weil der Säugling nach einer Schreiphase nicht einfach einschläft, auch wenn er sehr müde scheint, da die Schlafhomöostase noch ungenügend oder gar nicht entwickelt ist.

\section{Korrespondenzadresse}

PD Dr. O. Jenni

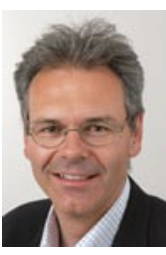

Abteilung Entwicklungspädiatrie, Universitätskinderkliniken Zürich Steinwiesstraße 75, 8032 Zürich Schweiz

Oskar.Jenni@kispi.uzh.ch

Interessenkonflikt. Der korrespondierende Autor gibt an, dass kein Interessenkonflikt besteht.

\section{Literatur}

1. Barr RG (1990) The normal crying curve: what do we really know? Dev Med Child Neurol 32(4):356-362

2. Barr RG (1993) Normality: a clinically useless concept. The case of infant crying and colic. J Dev Behav Pediatr 14(4):264-270

3. Barr RG, Konner M, Bakeman R, Adamson L (1991) Crying in!Kung San infants: a test of the cultural specificity hypothesis. Dev Med Child Neurol 33(7):601-610
4. Bensel J, Haug-Schnabel G (2003) Exzessives Schreien. In: Keller $\mathrm{H}$ (Hrsg) Handbuch der Kleinkindforschung. Huber, Bern, S 1195-1241

5. Borbély AA (1982) A two process model of sleep regulation. Hum Neurobiol 1(3):195-204

6. Brazelton TB (1962) Crying in infancy. Pediatrics 29:579-588

7. Dijk DJ, Czeisler CA (1995) Contribution of the circadian pacemaker and the sleep homeostat to sleep propensity, sleep structure, electroencephalographic slow waves, and sleep spindle activity in humans. J Neurosci 15(5):3526-3538

8. Edgar DM, Dement WC, Fuller CA (1993) Effect of SCN lesions on sleep in squirrel monkeys: evidence for opponent processes in sleep-wake regulation. J Neurosci 13(3):1065-1079

9. Emde RN, Gaensbauer TJ, Harmon RJ (1976) Emotional expression in infancy: a biobehavioral study. Psychological issues, a monograph series 10(37). International Universities Press, New York

10. Glotzbach SF, Edgar DM, Boeddiker M, Ariagno RL (1994) Biological rhythmicity in normal infants during the first 3 months of life. Pediatrics 94(4):482-488

11. Jenni OG, Benz C (2007) Schlafstörungen im Kindesalter. Pädiatr Up2date 4:309-333

12. Jenni OG, LeBourgeois MK (2006) Understanding sleepwake behavior and sleep disorders in children: the value of a model. Curr Opin Psychiatry 19(3):282-287

13. Jenni OG, Borbély AA, Achermann A (2004) Development of the nocturnal sleep electroencephalogram in human infants. Am J Physiol 286:R528-R538

14. Jenni OG, DeBoer T, Achermann P (2006) Development of the 24 -h rest-activity pattern in human infants. Infant Behav Dev 29:143-152

15. Kries R von (2006) Exzessives Schreien bei jungen Säug lingen: Definitionen, Häufigkeiten, Risikofaktoren, natürlicher Verlauf und Prognose. Kinderärztl Prax 77:84-88

16. Lehtonen L, Gormally S, Barr RG (2000) "Clinical pies" for etiology and outcome in infants presenting with early increased crying. In: Barr RG, Hopkins B, Green JA (eds) Crying as a sign, a symptom, and a signal. Mac Keith Press, London, pp 67-95

17. Löhr B, Siegmund R (1999) Ultradian and circadian rhythms of sleep-wake and food-intake behavior during early infancy. Chronobiol Int 16(2):129-148

18. Papoušek M, Hofacker N von (1998) Persistent crying in early infancy: a non-trivial condition of risk for the developing mother-infant relationship. Child Care Health Dev 24(5):395-424

19. Papoušek M, Schieche M, Wurmser H (2004) Regulationsstörungen in der frühen Kindheit. Huber, Bern

20. Porkka-Heiskanen T, Alanko L, Kalinchuk A, Stenberg D (1997) Adenosine: a mediator of the sleep-inducing effects of prolonged wakefulness. Science 276(5316):1265-1268

21. Rao MR, Brenner RA, Schistermann EF et al (2004) Long term cognitive development in children with prolonged crying. Arch Dis Child 89(11):989-992

22. Rivkees SA (2003) Developing circadian rhythmicity in infants. Pediatrics 112(2):373-381

23. St. James-Roberts I (2007) Infant crying and sleeping helping parents to prevent and manage problems. In: Jenni OG, Carskadon MA (eds) Sleep in children and adolescents. Elsevier Saunders, Philadelphia, pp 363-375

24. St. James-Roberts I, Halil T (1991) Infant crying patterns in the first year: normal community and clinical findings. J Child Psychol Psychiatry 32(6):951-968

25. Tononi G, Cirelli C (2006) Sleep function and synaptic homeostasis. Sleep Med Rev 10:49-62

26. Wessel MA, Cobb JC, Jackson EB et al (1954) Paroxysmal fussing in infancy, sometimes called colic. Pediatrics 14(5):421-435

27. Wolke D, Rizzo P, Woods S (2002) Persistent infant crying and hyperactivity problems in middle childhood. Pediatrics 109(6):1054-1060

28. Ziegler M, Wollwerth de Chuquisengo R, Papousek M (2004) Exzessives Schreien im frühen Säuglingsalter. In: Papousek M, Schieche M, Wurmser H (Hrgs) Regulationsstörungen in der frühen Kindheit. Huber, Bern, $S$ $111-143$ 